\title{
Sensorless Stabilization of Bounce Juggling
}

\author{
Renaud Ronsse, Philippe Lefèvre, and Rodolphe Sepulchre, Member, IEEE
}

\begin{abstract}
The paper studies the properties of a sinusoidally vibrating wedge billiard as a model for 2 -D bounce juggling. It is shown that some periodic orbits that are unstable in the elastic fixed wedge become exponentially stable in the nonelastic vibrating wedge. These orbits are linked with certain classical juggling patterns, providing an interesting benchmark for the study of the frequency-locking properties in human rhythmic tasks. Experimental results on sensorless stabilization of juggling patterns are described.
\end{abstract}

Index Terms-Billiard, discrete time, feedback, impact control, juggling, rhythm, sensorless, stabilization.

\section{INTRODUCTION}

$\mathbf{T}$ HE control of rhythmic tasks, routinely accomplished by most animals in spite of changing and perturbed environments, remains a puzzle in science and engineering. One issue of particular interest in this framework is the issue of feedback: how much sensory information is needed to perform a rhythmic task, and how is this information integrated to produce intermittent control actions? This issue reaches across a number of disciplines and seems relevant to rhythmic robotics. But it has received limited attention in the control community beyond the pioneering work of Koditschek and coworkers, initially in juggling robotics [1]-[3], and more recently in legged robotics [4].

This paper presents a somewhat extreme and contrived situation in which the rhythmic task is unstable in the absence of actuation, but stabilized with a control that uses no feedback. Our objective is by no means to deemphasize the role of feedback in the control of rhythmic tasks, but rather to emphasize that the issue of feedback deserves particular attention in the framework of intermittent control.

The rhythmic task studied in this paper is the juggling of the shower pattern (Fig. 1) in a planar wedge-billiard. This robotic

Manuscript received February 3, 2005; revised June 16, 2005. This paper was recommended for publication by Associate Editor K. Lynch and Editor H. Arai upon evaluation of the reviewers' comments. This work was supported in part by the Belgian Program on Interuniversity Attraction Poles initiated by the Belgian Federal Science Policy Office, in part by the Fonds National de la Recherche Scientifique; in part by the Fondation pour la Recherche Scientifique Médicale; and in part by an internal research grant (Fonds Spéciaux de Recherche) of the Université catholique de Louvain. This paper was presented in part at the 6th IFAC Symposium on Nonlinear Control Systems, Stuttgart, Germany, 2004

R. Ronsse and R. Sepulchre are with the Department of Electrical Engineering and Computer Science (Montefiore Institute), Université de Liège, B-4000 Liège, Belgium (e-mail: R.Ronsse@ulg.ac.be; R.Sepulchre@ulg.ac.be).

P. Lefèvre is with the Center for Systems Engineering and Applied Mechanics (CESAME), Université catholique de Louvain, B-1348 Louvain-la-Neuve, Belgium (e-mail: lefevre@csam.ucl.ac.be).

This paper has supplementary multimedia material available at http://ieeexplore.ieee.org, provided by the author. This pack contains two avi files illustrating the sensorless stabilization of impact juggling orbits with the "Wiper" robot. "perone.avi" displays a period-one motion and "pertwo.avi" a period-two motion. They have been recorded at the University of Liège, Belgium. This material is $8.4 \mathrm{MB}$ in size.

Digital Object Identifier 10.1109/TRO.2005.858860

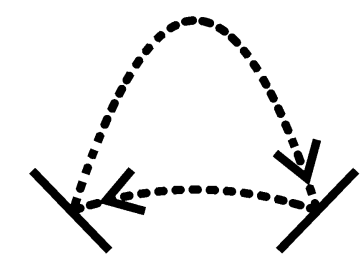

Fig. 1. Shower juggling pattern.

device is viewed as an idealization of a human juggler: the ball in a constant gravitational field undergoes collisions with two edges, acting as the juggler arms. We analyze the existence and stability of periodic orbits in the simplest mathematical model of this planar juggler. Our main result is to show that some exponentially unstable periodic orbits of the elastic model are stabilized in the nonelastic model with a simple control that uses no feedback measurement, hence, the name sensorless stabilization, and to provide an experimental validation of this result. The result somewhat contradicts the intuition that the stabilization of unstable steady states normally involves feedback. For the period-one orbit also discussed in this paper, the result has been previously observed by Schaal and Atkeson [5], at least numerically, who reported the planar juggler as one example of a rhythmic system that can be stabilized without feedback.

The control studied in this paper is a sinusoidal actuation of the edges, perhaps the simplest imitation of the fundamental cyclic motion of the hands of a juggler. The vibration frequency of the edges is the key parameter, since the stabilization mechanism rests on a synchronization (or frequency locking) between the controller and the tempo of the juggling pattern. The vibration amplitude is a critical control parameter, since the periodic orbits derived in this paper are stable for a restricted amplitude range.

An earlier analysis of this basic stabilization mechanism has been provided by the celebrated bouncing-ball model of Holmes [6], in which a ball bounces vertically on a sinusoidally vibrating table. In the elastic and unactuated model (fixed table), the ball continually bounces at the same height, depending on the initial condition, yielding a continuum of marginally stable periodic orbits in the state space. Sinusoidal actuation at the right frequency isolates and exponentially stabilizes one particular orbit in the nonelastic model, creating a stable limit cycle. This simple frequency-locking phenomenon is closely related to the stabilization mechanism described in this paper. In fact, a cartoon model of our 2-D juggler, studied in Section IV, precisely decouples into two 1-D bouncing balls. In this sense, most stability results in this paper can be understood as a persistence of this stabilization mechanism in nearby configurations, including configurations where the stabilized orbits are exponentially unstable in the elastic unactuated model (fixed wedge). 
The bouncing-ball model of Holmes has not only become a celebrated case study in nonlinear dynamics [6]-[8], but has also motivated recent studies of one-hand vertical juggling by Sternad et al. [9]-[11]. These studies suggest the tendency of humans to exploit stable sensorless strategies in order to alleviate or relax the need for sensory feedback information. The persistence of stable sensorless strategies in the planar (2-D) juggler model studied in this paper offers new opportunities to study the role of feedback in human tasks that require interlimb coordination and sensory information selection. Many earlier studies in rhythmic tasks have focused on juggling as a particularly relevant benchmark that requires a moderate amount of learning, and which is amenable to mathematical modeling and to experimental investigations, both in robotics and with human subjects [12], [13]. The important role of learning has been emphasized by Beek and coworkers [13], [14]. Feedback controlled planar devices that juggle a puck vertically have been developed and studied by Buehler et al. [1]-[3], by Lynch and Black [15], and by Zavala-Rio and Brogliato [16]. The role of feedback in a rhythmic task is investigated by Jin and Zacksenhouse (see [17] and related works) for yo-yo playing. It is open-loop unstable and requires phase-locking feedback to stabilize. They exploit the rhythmical feature of the task to design controllers with low-rate (discrete) feedback. In ongoing work, we use the planar device described in this paper for feedback control of the shower [18], [19], as well as for investigations on human bounce juggling.

The paper is organized as follows. The dynamic model of our bounce juggler is presented in Section II. Period-one and period-two orbits are characterized in Section III, and their stability is studied in the unactuated (fixed) wedge. In Section IV, we derive the steady-state periodic orbits of the sinusoidally actuated wedge-billiard. Considering a cartoon model of the vibrating wedge, this emphasizes the stabilization mechanism in analogy with the bouncing-ball dynamics. This analysis is extended in Section V to a model that takes into account further coupling phenomena in the rotating wedge implemented in the lab. Our experimental setup is described in Section VI, which contains a comparison between theoretical predictions and experimental results. Conclusions are presented in Section VII.

\section{A Bounce JugGLer Model}

The dynamical system studied in this paper is an idealization of a human juggler. We consider a motion restricted to a plane under a constant gravitational field $\mathbf{g}$ (with $|\mathrm{g}|=g$ ). The juggled ball undergoes collisions with two edges, which act as the juggler arms (see Fig. 2). In contrast with human juggler, the impacts between the "hands" and the ball are supposed to be instantaneous, hence the name impact (or bounce) juggling. This paper focuses on patterns involving one ball.

Our model is a control version of the model introduced in [20], and was first presented in [18] in order to study several closed-loop control laws to stabilize impact juggling patterns. The 4-D wedge-billiard dynamics are studied via the 3-D discrete Poincaré map relating the state from one impact to the next one, the ball motion between two impacts being parabolic (a ballistic flight in a constant gravitational field $\mathbf{g})$. Let $\left(\mathbf{e}_{r}, \mathbf{e}_{n}\right)$ be an orthonormal frame attached to the fixed point $O$ with $\mathbf{e}_{r}$ aligned with the ball position vector $\mathbf{r}=r \mathbf{e}_{r}$. The ball is assumed to be a unit mass point; let $\mathbf{v}=v_{r} \mathbf{e}_{r}+v_{n} \mathbf{e}_{n}$ denote

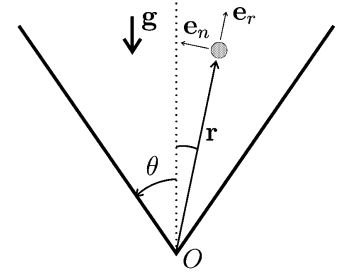

Fig. 2. Wedge-billiard.

its velocity. Therefore, the discrete state vector denotes the state of the ball at the impacts. This state being discontinuous at the impacts, we choose the postimpact values to make up the state vector as a convention

$$
\mathbf{x}[k] \equiv\left(\begin{array}{c}
V_{r}[k] \\
\left|V_{n}\right|[k] \\
R[k]
\end{array}\right)=\left(\begin{array}{c}
V_{r}^{+}(t[k]) \\
\left|V_{n}^{+}\right|(t[k]) \\
R^{+}(t[k])
\end{array}\right)
$$

where $V_{r}=\left(v_{r} / \cos \theta\right), V_{n}=\left(v_{n} / \sin \theta\right)$, and $R=(r / \cos \theta)$ denote the state of the ball, and the $\bullet^{+}[k]$ notations denote the postimpact values, evaluated at impact time $t[k]$. The corresponding preimpact values are denoted $\bullet^{-}[k]$. We consider the absolute value of the normal velocity, the wedge-billiard being symmetric with respect to its bissecting line.

The discrete wedge-billiard map is the composition of an impact rule and a parabolic flight map. The impact rule $\mathcal{I}$ adopted is derived from the Newton's impact law: the normal velocity is reversed, proportionally to a coefficient of restitution e modeling the energy dissipation at impact $(0 \leq e<1)$, while the tangential velocity is conserved. This paper focuses on solutions implying an actuation of the edges in order to feed some energy to the ball at each impact. This control input is captured in the impact rule, where the normal velocity expression is expressed relative to the edge velocity

$$
V_{n}[k]-\dot{s}[k]=-e\left(V_{n}^{-}[k]-\dot{s}[k]\right)
$$

where $\dot{s}[k]=\dot{s}(t[k])=(\dot{\tilde{s}}(t[k]) / \sin \theta)$, and $\dot{\tilde{s}}(t[k])$ denotes the edge velocity at the impact time $t[k]$. The impact rule $\mathcal{I}$ is then

$$
\begin{aligned}
V_{r}[k] & =V_{r}^{-}[k] \\
V_{n}[k] & =-e V_{n}^{-}[k]+(1+e) \dot{s}[k] .
\end{aligned}
$$

Assuming that the change in the edges position can be neglected to compute the ball flight map (this assumption rests basically on a small amplitude actuation of the edges; see the discussion in Section V), the flight map is the velocity and position update of the ball integrated through a flight between two impacts on the unactuated wedge-billiard. Two different flight maps must be considered, whether these impacts occur on the same edge or not. These flight maps have been derived in [18]. The rest of this paper focusing only on solutions and stability properties, where the ball hits the edges alternately, the wedge-billiard map is, therefore, the composition of the impact rule $\mathcal{I}$ (1) and the second flight map [20], [18]

$$
\begin{aligned}
& V_{r}^{-}[k+1]=\left|V_{n}\right|[k]-V_{r}[k]-\left|V_{n}^{-}\right|[k+1] \\
& \left|V_{n}^{-}\right|[k+1] \\
& \quad=\sqrt{\left(\frac{2 V_{r}[k]+\left(\alpha^{2}-1\right)\left|V_{n}\right|[k]}{1+\alpha^{2}}\right)^{2}+\frac{4 g}{1+\alpha^{2}} R[k]}
\end{aligned}
$$


with

$$
\alpha=\tan \theta
$$

One obtains the discrete billiard map $\mathcal{B}$

$$
\begin{aligned}
V_{r}[k+1]= & \left|V_{n}\right|[k]-V_{r}[k]-\left|V_{n}^{-}\right|[k+1] \\
V_{n}[k+1]= & -e\left|V_{n}^{-}\right|[k+1] \operatorname{sign}\left(V_{n}[k]\right) \\
& +(1+e) \dot{s}[k+1] \\
R[k+1]= & R[k]-\frac{1}{2 g}\left(V_{r}^{2}[k+1]-V_{r}^{2}[k]\right) \\
& -\frac{\alpha^{2}}{2 g}\left(\left|V_{n}^{-}\right|^{2}[k+1]-V_{n}^{2}[k]\right) .
\end{aligned}
$$

The position update of (3) derives from the energy expression

$$
E[k]=\frac{1}{1+\alpha^{2}}\left(\frac{1}{2} V_{r}^{2}[k]+\frac{\alpha^{2}}{2} V_{n}^{2}[k]+g R[k]\right)
$$

and the conservation of energy through the flight implies $E^{-}[k+1]=E[k]$.

For later reference, one also notes the flight-time equation given by

$$
\begin{aligned}
\Delta t[k] & \equiv t[k+1]-t[k] \\
& =\frac{1}{g}\left(\left|V_{n}^{-}\right|[k+1]+\frac{\left(\alpha^{2}-1\right)\left|V_{n}\right|[k]+2 V_{r}[k]}{1+\alpha^{2}}\right) .
\end{aligned}
$$

\section{ENERGy BALANCE OF THE SteAdy-StATE SOlutions}

One of the most common juggling patterns is called the shower. It involves a circle-shaped trip of several balls between the juggler hands (Fig. 1). This section will describe and analyze a periodic solution of the model (3), which is very close to the shower pattern. The ball(s) trip is also a composition of two trajectories between the edges: a high and a low toss. In the state space of the discrete dynamical system (3), this periodic solution is characterized by a periodic jump between two fixed points, and is then called a period-two solution (or orbit).

The first part of this section describes a degenerate case of the period-two orbits where both flight times are equal. In this case, the ball follows a round trip between the edges on the same trajectory. This solution is characterized by a fixed point in the state space, and is called the period-one solution. The general period-two solution of the wedge-billiard will be investigated in Section III-B. Stability properties of these solutions in the unactuated elastic wedge will be also investigated.

\section{A. Period-One Orbit}

This solution is a round trip of the ball between the edges, both trajectories being exactly the same [Fig. 3(a)]. Due to its symmetry, this periodic motion is characterized by a unique energy level $E^{*}$. The radial velocity $V_{r}$ and the position $R$ being conserved at impacts, the conservation of energy implies the conservation of the square normal velocity

$$
\left(V_{n}^{*}\right)^{2}=\left(V_{n}^{-*}\right)^{2}
$$

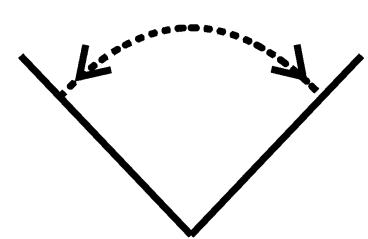

(a)

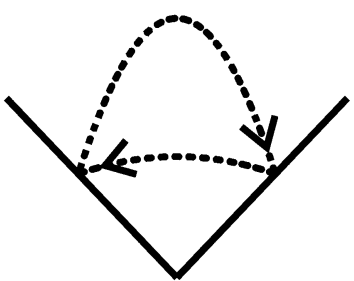

(b)
Fig. 3. Two periodic orbits of the wedge-billiard. (a) Period-one solution. (b) Period-two solution.

Using (6), we obtain, in the steady-state solution of (3)

$$
\begin{aligned}
V_{r}^{*} & =\left|V_{n}^{*}\right|-V_{r}^{*}-\left|V_{n}^{-*}\right| \\
\left|V_{n}^{*}\right| & =e\left|V_{n}^{-*}\right|+(1+e)\left|\dot{s}^{*}\right|
\end{aligned}
$$

which implies $V_{r}^{*}=0$. These results have a direct geometrical interpretation on Fig. 3(a). At the impacts, the radial velocity must be zero, and the normal velocity must be exactly reversed, for this steady-state motion. The steady-state edges velocity derives from $(8)$

$$
\left|\dot{s}^{*}\right|=\frac{1-e}{1+e}\left|V_{n}^{*}\right| .
$$

Using (4), (5), and (2), the fixed point of (3) is conveniently parametrized by the energy $E^{*}$

$$
\begin{aligned}
\left|V_{n}^{*}\right| & =\frac{1+\alpha^{2}}{\alpha} \sqrt{\frac{2 E^{*}}{3+\alpha^{2}}} \\
R^{*} & =\frac{2\left(1+\alpha^{2}\right)}{g\left(3+\alpha^{2}\right)} E^{*} \\
\Delta t^{*} & =\frac{2 \alpha}{g} \sqrt{\frac{2 E^{*}}{3+\alpha^{2}}} .
\end{aligned}
$$

If the edges are elastic $(e=1)$, no energy supply is needed to sustain the period-one motion (see (9): $\left|\dot{s}^{*}\right|=0$ ). This could be also simulated from a nonelastic wedge-billiard if the edges compensate for the energy dissipation at each impact $|\dot{s}|[k+$ $1]=(1-e) /(1+e)\left|V_{n}^{-}\right|[k+1]$.

Proposition 1 (see [20]): For every $\theta \in\left(0^{\circ}, 90^{\circ}\right)$ and for every energy level $E^{*}$, the wedge-billiard possesses a unique period-one orbit, determined by the fixed point (10)-(12) of model (3). This orbit is marginally stable if $\theta<45^{\circ}(\alpha<1)$, unstable if $\theta=45^{\circ}(\alpha=1)$, and exponentially unstable if $\theta>45^{\circ}(\alpha>1)$.

Proof: Stability of the period-one orbits is investigated via the Jacobian linearization of (3) at the fixed point (10)-(12), which gives (13)

$$
\begin{aligned}
& \left(\begin{array}{c}
\delta V_{r}[k+1] \\
\delta\left|V_{n}\right|[k+1] \\
\frac{g}{\left|V_{n}^{*}\right|} \delta R[k+1]
\end{array}\right) \\
& =\left(\begin{array}{ccc}
\frac{1-4 \alpha^{2}-\alpha^{4}}{\left(1+\alpha^{2}\right)^{2}} & \frac{4 \alpha^{2}}{\left(1+\alpha^{2}\right)^{2}} & \frac{-2}{1+\alpha^{2}} \\
\frac{2\left(\alpha^{2}-1\right)}{\left(1+\alpha^{2}\right)^{2}} & \frac{\left(\alpha^{2}-1\right)^{2}}{\left(1+\alpha^{2}\right)^{2}} & \frac{2}{1+\alpha^{2}} \\
\frac{2 \alpha^{2}\left(1-\alpha^{2}\right)}{\left(1+\alpha^{2}\right)^{2}} & \frac{4 \alpha^{4}}{\left(1+\alpha^{2}\right)^{2}} & \frac{1-\alpha^{2}}{1+\alpha^{2}}
\end{array}\right)\left(\begin{array}{c}
\delta V_{r}[k] \\
\delta\left|V_{n}\right|[k] \\
\frac{g}{\left|V_{n}^{*}\right|} \delta R[k]
\end{array}\right) .
\end{aligned}
$$


The eigenvalues of this Jacobian matrix are

$$
\lambda_{1,2,3}=1, \frac{1-4 \alpha^{2}-\alpha^{4} \pm 2 \alpha \sqrt{\left(\alpha^{2}-1\right)\left(\alpha^{2}+3\right)}}{\left(1+\alpha^{2}\right)^{2}} .
$$

One unitary eigenvalue $\left(\lambda_{1}=1\right)$ is associated with the conservation of energy. The remaining two eigenvalues $\left(\lambda_{2}\right.$ and $\lambda_{3}$ ) lie on the unitary circle for $\alpha<1$, and it can be shown that the period-one solution is marginally stable in this case. In the state-space plane of the elastic wedge-billiard, the period-one fixed point is surrounded by a continuum of closed orbits that correspond to quasi-periodic solutions [20]. When the wedge is a right angle $(\alpha=1), \lambda_{2}=\lambda_{3}=-1$ : the map $\mathcal{B}$ therefore has an eigenvalue of algebraic multiplicity 2 on the unit circle. The unactuated wedge-billiard dynamics becoming linear in that case (see [18]), the period-one orbits are unstable. For $\alpha>1$, the two eigenvalues are real, one of them being outside the unitary circle; the period-one solution is, therefore, exponentially unstable.

\section{B. Period-Two Orbit}

Period-two orbits model the shower juggling pattern presented in Fig. 1. The round trip of the ball between the edges is now characterized by two different trajectories, depending on the direction of the ball. Fig. 3(b) is an example of a period-two solution. It is easily connected with the shower pattern. This solution will be characterized by two parameters $E^{r_{*}}$ and $E^{l_{*}}$, associated with the ball energy on each trajectory, assuming arbitrarily that $\bullet^{r_{*}}$ characterizes the right-edge impacts, and $\bullet \boldsymbol{l}_{*}$, the left-edge impacts. A positive edge velocity corresponds to a counterclockwise motion. The period-one orbit is a degenerate case of the period-two orbits for which $E^{r_{*}}=E^{l_{*}}$.

A period-two solution is characterized by two points in the state space, say $\mathbf{x}^{r_{*}}$ and $\mathbf{x}^{l_{*}}$; these points ought to be fixed points of $\mathcal{B}^{2}=\mathcal{B} \circ \mathcal{B}$. The position and the radial velocity being conserved at impact, the global energy balance of the period-two solution requires this time

$$
\left(V_{n}^{r_{*}}\right)^{2}-\left(V_{n}^{-r_{*}}\right)^{2}=-\left(\left(V_{n}^{l_{*}}\right)^{2}-\left(V_{n}^{-l_{r}}\right)^{2}\right)
$$

A possible loss of energy on one edge has to be compensated on the other one.

Eliminating the radial velocities $V_{r}{ }^{r_{*}}$ and $V_{r}{ }^{l_{*}}$ from their update equations

$$
\begin{gathered}
V_{r}^{l_{*}}=\left|V_{n}^{r_{*}}\right|-V_{r}^{r_{*}}-\left|V_{n}^{-l_{*}}\right| \\
V_{r}^{r_{*}}=\left|V_{n}^{l_{*}}\right|-V_{r}^{l_{*}}-\left|V_{n}^{-r_{*}}\right|
\end{gathered}
$$

we find another relation between the normal velocities

$$
\left|V_{n}^{r_{*}}\right|-\left|V_{n}^{-l_{*}}\right|=\left|V_{n}^{l_{*}}\right|-\left|V_{n}^{-r_{*}}\right| \text {. }
$$

Equations (15) and (18) yield

$$
\left|V_{n}^{r_{*}}\right|+\left|V_{n}^{-l_{*}}\right|=-\left(\left|V_{n}^{l_{*}}\right|+\left|V_{n}^{-r_{*}}\right|\right) .
$$

Thanks to (18) and (19), one finds $\left|V_{n}^{-l_{*}}\right|=-\left|V_{n}^{l_{*}}\right|$ and $\left|V_{n}^{-r_{*}}\right|=-\left|V_{n}^{r_{*}}\right|$. These four variables being nonnegative, (18) must be equal to zero to be satisfied, such as (15)

$$
\begin{aligned}
& \left|V_{n}^{-l_{*}}\right|=\left|V_{n}^{r_{*}}\right| \\
& \left|V_{n}^{-r_{*}}\right|=\left|V_{n}^{l_{*}} .\right|
\end{aligned}
$$

The radial velocity and the impact position should then satisfy [see (16) and (3)]

$$
\begin{aligned}
& V_{r}^{l_{*}}=-V_{r}^{r_{*}} \\
& R^{r_{*}}=R^{l_{*}}=R^{*} .
\end{aligned}
$$

Geometrically, the period-two solutions are therefore characterized by two symmetrical parabolas. These parabolas reach their highest point (zenith) on the bissecting line of the wedge.

Introducing the normal velocity conservation (20) in (3), we obtain the steady-state relations

$$
\begin{aligned}
& \left|V_{n}{ }^{l_{*}}\right|=e\left|V_{n}{ }^{r_{*}}\right|-(1+e) \dot{s}^{l_{*}} \\
& \left|V_{n}{ }^{r_{*}}\right|=e\left|V_{n}{ }^{l_{*}}\right|+(1+e) \dot{s}^{r_{*}} .
\end{aligned}
$$

Equations (23) provide the steady-state edges velocities

$$
\begin{aligned}
\dot{s}^{r_{*}} & =\frac{\left|V_{n}^{r_{*}}\right|-e\left|V_{n}^{l_{*}}\right|}{1+e} \\
\dot{s}^{l_{*}} & =\frac{e\left|V_{n}^{r_{*}}\right|-\left|V_{n}^{l_{*}}\right|}{1+e} .
\end{aligned}
$$

Injecting (20) in the definitions of $\left|V_{n}^{-}\right|$, we find

$$
\begin{gathered}
\left(V_{n}^{r_{*}}\right)^{2}=\left(\frac{2 V_{r}^{r_{*}}+\left(\alpha^{2}-1\right)\left|V_{n}^{r_{*}}\right|}{1+\alpha^{2}}\right)^{2}+\frac{4 g}{1+\alpha^{2}} R^{*} \\
\left(V_{n}^{l_{*}}\right)^{2}=\left(\frac{2 V_{r}^{l_{*}}+\left(\alpha^{2}-1\right)\left|V_{n} l_{*}\right|}{1+\alpha^{2}}\right)^{2}+\frac{4 g}{1+\alpha^{2}} R^{*}
\end{gathered}
$$

which implies, taking (21) into account

$$
V_{r}^{r_{*}}=\frac{\alpha^{2}}{\alpha^{2}-1}\left(\left|V_{n}^{r_{*}}\right|-\left|V_{n}^{l_{*}}\right|\right)=-V_{r}^{l_{*}} .
$$

The impact position and the energy levels are derived from (25), (27), (4), and (28)

$$
\begin{aligned}
R^{*} & =\frac{\alpha^{2}\left(\left(1+\alpha^{2}\right)^{2}\left|V_{n}^{r_{*}}\right|\left|V_{n}^{l_{*}}\right|-\alpha^{2}\left(\left|V_{n}^{r_{*}}\right|+\left|V_{n}^{l_{*}}\right|\right)^{2}\right)}{g\left(1+\alpha^{2}\right)\left(\alpha^{2}-1\right)^{2}} \\
E^{r_{*}} & =\frac{\alpha^{2}\left(\left(\alpha^{4}+\alpha^{2}-1\right)\left(V_{n}^{r_{*}}\right)^{2}+\alpha^{2}\left(V_{n}^{l_{*}}\right)^{2}-2\left|V_{n}^{r_{*}}\right|\left|V_{n}^{l_{*}}\right|\right)}{2\left(\alpha^{2}-1\right)\left(1+\alpha^{2}\right)^{2}} \\
E^{l_{*}} & =\frac{\alpha^{2}\left(\left(\alpha^{4}+\alpha^{2}-1\right)\left(V_{n}^{l_{*}}\right)^{2}+\alpha^{2}\left(V_{n}^{r_{*}}\right)^{2}-2\left|V_{n}^{r_{*}}\right|\left|V_{n}^{l_{*}}\right|\right)}{2\left(\alpha^{2}-1\right)\left(1+\alpha^{2}\right)^{2}}
\end{aligned}
$$

If the energy levels denote the two parameters of the period-two solution, (29) must be inversed to find the normal velocities that can be replaced in the solution equations.

The flight times are derived from (5)

$$
\begin{aligned}
\Delta t^{r_{*}} & =\frac{2 \alpha^{2}\left(\alpha^{2}\left|V_{n}^{r_{*}}\right|-\left|V_{n}^{l_{*}}\right|\right)}{g\left(\alpha^{4}-1\right)} \\
\Delta t^{l_{*}} & =\frac{2 \alpha^{2}\left(\alpha^{2}\left|V_{n}^{l_{*}}\right|-\left|V_{n}^{r_{*}}\right|\right)}{g\left(\alpha^{4}-1\right)} .
\end{aligned}
$$

These relationships correspond to the period-one solution (10)-(12) if $E^{r_{*}}=E^{l_{*}}\left(\left|V_{n}^{r_{*}}\right|=\left|V_{n}^{l_{*}}\right|\right)$, with the equation shown at the bottom of the next page. 
An elastic wedge can be simulated from nonelastic edges by adding $(1-e / 1+e)\left|V_{n}^{-}\right|[k+1]$ to the edges velocity.

Proposition 2: Period-two orbits exist in the unactuated elastic wedge-billiard only for $\theta=45^{\circ}$. They are uniquely defined by their energy level $E^{*}$ and their impact radial velocity $V_{r}$, and are unstable. For every $\theta \in\left(0^{\circ}, 90^{\circ}\right)$ and for every pair $\left(E^{r_{*}}, E^{l_{*}}\right)$, the actuated elastic wedge-billiard possesses a unique period-two orbit determined by the fixed point of $\mathcal{B}^{2}=\mathcal{B} \circ \mathcal{B}$ (3). If $\theta<45^{\circ}$, this orbit is either marginally stable or exponentially unstable, depending on the energy difference $E^{r_{*}}-E^{l_{*}}$. It is unstable if $\theta=45^{\circ}$, and exponentially unstable if $\theta>45^{\circ}$.

Proof: First, we derive the period-two orbits in the particular case $\theta=45^{\circ}(\alpha=1)$, because several equations previously derived become singular. This special configuration is called the square wedge-billiard, the edges forming a right angle. In this case, (21), (25), and (26) force the normal velocities to be equal

$$
\left|V_{n}^{r_{*}}\right|=\left|V_{n}{ }^{l_{*}}\right|=\left|V_{n}^{*}\right|=\left|V_{n}^{-r_{*}}\right|=\left|V_{n}^{-l_{*}}\right|
$$

so that each trajectory has the same energy $E^{r_{*}}=E^{l_{*}}=E^{*}$. In this case, the second parameter of the period-two orbit is the radial velocity $V_{r}^{*}$, since (27) is undetermined when $\alpha=1$.

The period-two solution of the square wedge-billiard is then derived from (4), (2), and (5) with $\alpha=1$

$$
\begin{aligned}
\left|V_{n}^{*}\right| & =\sqrt{2 E^{*}} \\
R^{*} & =\frac{1}{2 g}\left(2 E^{*}-\left(V_{r}^{*}\right)^{2}\right) \\
\Delta t^{r_{*}}, \Delta t^{l_{*}} & =\frac{\sqrt{2 E^{*}} \pm\left|V_{r}^{*}\right|}{g} .
\end{aligned}
$$

From (24), we have

$$
\dot{s}^{r_{*}}=-\dot{s}^{l_{*}}=\frac{\left|V_{n}^{r_{*}}\right|-\left|V_{n}^{l_{*}}\right|}{2} .
$$

The steady-state edges velocities $\dot{s}^{r_{*}}$ and $\dot{s}^{l_{*}}$ are therefore equal to zero only if $\left|V_{n}^{r_{*}}\right|=\left|V_{n}^{l_{*}}\right|$, i.e., in the square configuration. Period-two orbits exist in the unactuated elastic wedge-billiard only in that configuration. Period-two orbits exist with any other wedge angle but require an actuation of the edges, according to (35).

Stability of these orbits is studied via the linearization of $\mathcal{B}^{2}=\mathcal{B} \circ \mathcal{B}(3)$ around its fixed point. We find the matrix

$$
\begin{aligned}
& \left(\begin{array}{c}
\delta V_{r}[k+2] \\
\frac{\delta\left|V_{n}\right|[k+2]}{\sqrt{\left|V_{n}{ }^{r_{*}}\right|\left|V_{n} l_{*}\right|}} \delta R[k+2]
\end{array}\right) \\
& =\mathcal{M}_{2}\left(\begin{array}{c}
\delta V_{r}[k+1] \\
\delta\left|V_{n}\right|[k+1] \\
\frac{g}{\sqrt{\left|V_{n} r_{*}\right| \mid V_{n}{ }^{l_{*} \mid}}} \delta R[k+1]
\end{array}\right)
\end{aligned}
$$

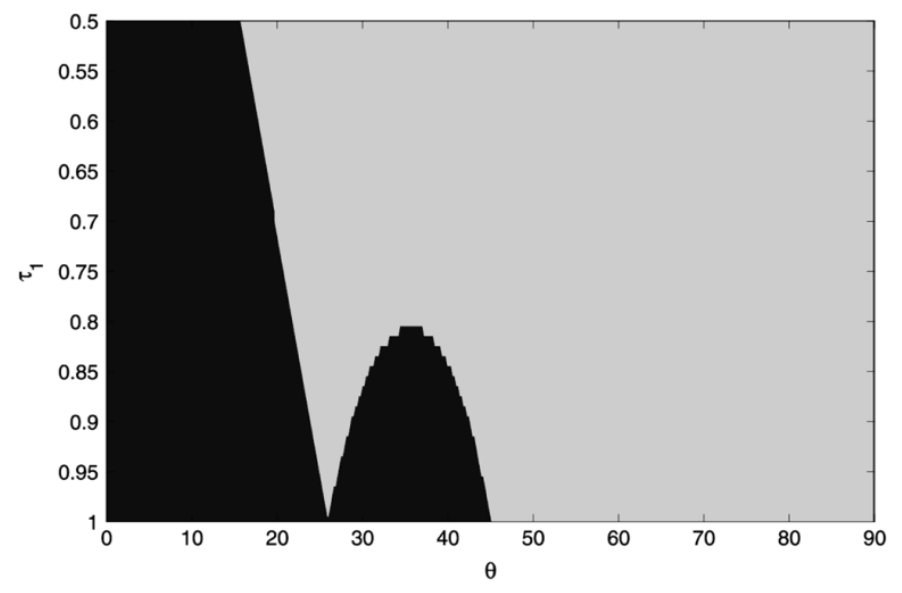

Fig. 4. Stability regions of the period-two solution: marginal stability (black) and exponential instability (grey).

$$
=\mathcal{M}_{2} \mathcal{M}_{1}\left(\begin{array}{c}
\delta V_{r}[k] \\
\frac{\delta\left|V_{n}\right|[k]}{\sqrt{\left|V_{n}{ }^{r_{*}}\right|\left|V_{n} l_{*}\right|}} \delta R[k]
\end{array}\right)
$$

with $\gamma_{i}=\left(\left(1-\tau_{i}\right) /\left(1-\alpha^{2}\right)\right)$ for $i=1,2$, and $\tau_{1}=\left(\left|V_{n}^{r_{*}}\right| /\left|V_{n}^{l_{*}}\right|\right)=\left(1 / \tau_{2}\right)$ denoting the normal velocity ratio. These parameters capture the energy difference between both parabolas, and have been defined as such only for computational convenience.

The eigenvalues of (36) were numerically computed, depending on $\alpha$ and $\tau_{1}$. Fig. 4 depicts the stability regions of the period-two solution in the parameter space $\left(\theta, \tau_{1}\right)$. As in the period-one stability analysis, one of the eigenvalues is always equal to one, the energy being conserved over two impacts in the period-two orbit. The two remaining eigenvalues either are complex and lie on the unitary circle (black zone, marginal stability) or are real, at least one of them being outside the unitary circle (grey zone, instability).

Similar to what was observed for the period-one orbit, ${ }^{1}$ Fig. 4 shows that all period-two orbits are exponentially unstable for $\theta>45^{\circ}$. In contrast, marginally stable orbits coexist with unstable orbits for $\theta<45^{\circ}$, depending on the energy difference $\left|E^{r_{*}}-E^{l_{*}}\right|$ via the ratio $\tau_{1}$. Finally, for $\theta=45^{\circ},\left|V_{n}^{r_{*}}\right|=\left|V_{n}^{l_{*}}\right|$ induces $\tau_{1}=\tau_{2}=1$. The three eigenvalues of $\mathcal{M}_{2} \mathcal{M}_{1}$ are equal to one. Because the dynamics become linear (see [18]), then the period-two orbits are unstable.

It is of interest to observe the unstable behavior of all period-two orbits for a particular value of $\theta$ about $26^{\circ}$. This value corresponds to $\left(\left(1-\alpha^{2}\right) /\left(1+\alpha^{2}\right)^{2}\right)=(1 / 2)$ (i.e., $\alpha=$ $\sqrt{\sqrt{5}-2})$, in which case, two eigenvalues of the linearized

${ }^{1}$ Note that the period-one eigenvalues (14) lie on a "slice" of Fig. 4, for $\tau_{1}=$ $\tau_{2}=1\left(\gamma_{1}=\gamma_{2}=0\right)$. In that case, $\mathcal{M}_{1}$ and $\mathcal{M}_{2}$ are equal to (13).

$$
\mathcal{M}_{i}=\left(\begin{array}{ccc}
\frac{1-4 \alpha^{2}-\alpha^{4}+4 \alpha^{2} \gamma_{i}}{\left(1+\alpha^{2}\right)^{2}} & \frac{2 \alpha^{2}\left(1+\tau_{i}\right)}{\left(1+\alpha^{2}\right)^{2}} & \frac{-2 \sqrt{\tau_{i}}}{1+\alpha^{2}} \\
\frac{\alpha^{4}+1}{\tau_{i}}-2 \alpha^{2} & \frac{\alpha^{4}+1}{\tau_{i}}-2 \alpha^{2} & \frac{2}{\left(1+\alpha^{2}\right)^{2}} \\
\frac{2 \alpha^{2}\left(1-\alpha^{2}+\left(1+\alpha^{2}\right) \gamma_{i}+2 \alpha^{2} \gamma_{i}^{2}\right)}{\left(1+\alpha^{2}\right) \sqrt{\tau_{i}}} \\
\frac{2 \alpha^{4}\left(1+\alpha_{i}\right)\left(1+\gamma_{i}\right)}{\left(1+\alpha^{2}\right)^{2} \sqrt{\tau_{i}}} & \frac{1-\alpha^{2}-2 \alpha^{2} \gamma_{i}}{1+\alpha^{2}}
\end{array}\right)
$$


system (13) are equal to $\pm i$. The linearized system (36) corresponds to a double iteration of (13) when $\tau_{1}=1$, resulting in two eigenvalues equal to $( \pm i)^{2}=-1$. They split into two real eigenvalues, one of them outside the unit circle, when $\tau_{1} \neq 1$. The same critical value of $\theta$ causes uncontrollability of a linearized model of the elastic wedge-billiard which is controlled with one edge. For this particular aperture, the dynamics of the normal velocity, the radial velocity, and the energy decouple. At first order, the control input leaves each of these dynamics invariant [19].

\section{PERIODIC ORbits of THE Sinusoidally ACTUATED WEDGE-BILLIARD}

None of the periodic orbits studied in Section III are attractors; either the linearized system is marginally stable or unstable. In this section, we introduce how a simple periodic actuation of the wedge can isolate and stabilize one of the periodic orbits, characterized by its energy level(s) $\left(E^{*}\right.$ for a period-one orbit, or $E^{r_{*}}$ and $E^{l_{*}}$ for a period-two orbit).

The stabilization method presented in this section is based on harmonic motion of each edge. A simple sinusoidal actuation of the edges is the closest imitation of the fundamental cyclic motion of the juggler's hands, $A \sin (\omega t)$, where $A$ and $\omega$ are the vibration amplitude and pulsation.

The steady-state periodic orbits are first derived for the square configuration that turns out to bridge the well-known bouncingball dynamics [6] and the wedge dynamics.

\section{A. The Square Wedge-Billiard}

The steady-state velocity-energy relation of the square wedge-billiard (32) has exactly the same form as for a bouncing ball, ${ }^{2}$ emphasizing the decoupling of the square wedge. The dynamics along each edge can be viewed as a one-degree-of-freedom (1-DOF) independent bouncing-ball motion [18]. The parameter $V_{r}^{*}$ determines the phase shift between the two bouncing balls.

The steady-state regime is characterized by two frequencylocking relations between the ball and the wedge [21]

$$
\begin{aligned}
& (t[k+2]-t[k])^{*} \equiv \Delta t^{r_{*}}+\Delta t^{l_{*}}=n \frac{2 \pi}{\omega} \\
& (t[k+1]-t[k])^{*} \equiv \Delta t^{r_{*}}=(2 m-1) \frac{\pi}{\omega}
\end{aligned}
$$

where $(\cdot)^{*}$ denotes the steady-state solutions. These relations rest on the trivial assumption $m \leq n, m$ and $n$ being positive integers. Equation (37) expresses that the ball period is a multiple of the edge vibration period; this is the frequency-locking relation of each dynamics. Equation (38) expresses that the phase difference between two successive impacts must be equal to an odd multiple of the vibration half-frequency. As a convention, $m$ will be associated with the flight time between the right edge and the left edge, $\Delta t^{r_{*}}=(2 m-1)(\pi / \omega)$, while the flight time between the left edge and the right edge will be $\Delta t^{l_{*}}=(2 n-$ $2 m+1)(\pi / \omega)$ in the steady-state regime. Table I illustrates the

\footnotetext{
${ }^{2}$ Consider simply that the bouncing-ball dynamics refer to an exchange of a maximal potential energy $E^{*}$ (at the top point) and a maximal kinetic energy $\propto\left(V_{n}^{*}\right)^{2}$ (just before impact), i.e., $\left|V_{n}^{*}\right| \propto \sqrt{E^{*}}$.
}

TABLE I

Periodic ORbits For the SQUARE WedGe-BILliard. $(x: y)$ DENOTES THE Ratio Between Both Flight Times Where $x(y)$ Is Associated With the

\begin{tabular}{|c|c|c|c|c|c|}
\hline & $\mathrm{m}=1$ & $\mathrm{~m}=2$ & $\mathrm{~m}=3$ & $\mathrm{~m}=4$ & $\ldots$ \\
\hline $\mathrm{n}=1$ & & $\mathrm{X}$ & $X$ & $\mathrm{X}$ & \\
\hline $\mathrm{n}=2$ & & & $X$ & $\mathrm{X}$ & \\
\hline $\mathrm{n}=3$ & & & & $X$ & $\ldots$ \\
\hline $\mathrm{n}=4$ & & & & & \\
\hline & & & & & \\
\hline
\end{tabular}
FLIGHT FROM LEFT TO RIGHT (FROM RIGHT TO LEFT)

first periodic orbits for the vibrating square wedge-billiard and the ratios between the low-toss and the high-toss flight times for each of these orbits. A sustained steady-state shower pattern will be characterized exactly by the same ratios, $n$ denoting also the number of juggled balls. It is interesting to point out how the symmetry of the square wedge-billiard captures the symmetry of the juggler behavior. Beek and Lewbel [13] wrote a very accessible paper on the "scientific aspects of juggling," where they present a compact notation for juggling patterns. Site-swap notation represents the order in which props are thrown and caught in each cycle of the juggle, assuming throws happen on beats that are equally spaced in time, being the case both in most of the common juggling patterns, and in the square wedge-billiard. The site-swap notation of the three balls shower is simply " 51 ," where the 5 refers to the duration of the high toss and the 1 to the time needed to pass the ball from one hand to the other on the lower part of the arc. Each $(x: y)$ orbit presented in Table I will then be "site-swap" noted " $x y$."

Injecting the flight-time solutions (34) in (37) and (38), we obtain the steady-state velocities of the periodic orbits of the square wedge-billiard, while the energy $E^{*}$ and the impact position $R^{*}$ derive from (32) and (33)

$$
\begin{aligned}
E^{*} & =\frac{(n \pi g)^{2}}{2 \omega^{2}} \\
\left|V_{n}^{*}\right| & =\frac{n \pi g}{\omega} \\
V_{r}^{r_{*}} & =-\frac{(n-2 m+1) \pi g}{\omega}=-V_{r}^{l_{*}} \\
R^{*} & =\frac{g}{2}\left(\frac{\pi}{\omega}\right)^{2}(2 m-1)(2 n-2 m+1) .
\end{aligned}
$$

The steady-state energy is independent of $m$. The radial velocity naturally corresponds to the period-one solution $\left(V_{r}^{*}=0\right)$ when $n=2 m-1$ (see Table I).

Let us briefly consider a cartoon model of the actuated square wedge-billiard, i.e., a model with a sinusoidal actuation along the axis perpendicular to the edges (Fig. 5). In that case, the normal velocity is updated as $V_{n}[k+2]=e V_{n}[k]+(1+$ $e) \dot{s}[k+2]$, exhibiting the decoupling between the dynamics along each edge. The stability properties of the periodic orbits are immediately inherited from the decoupled dynamics, i.e., from the stability properties of a bouncing ball, which have been 


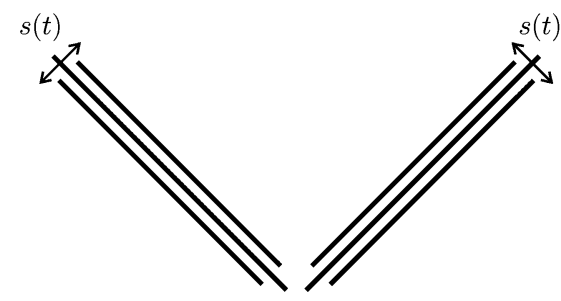

Fig. 5. Cartoon model of an actuated square wedge-billiard.

studied in [6]. A period-one motion is exponentially stable for the nonelastic bouncing ball if

$$
n \pi \frac{1-e}{1+e}<\frac{\sqrt{2} A \omega^{2}}{g}<\sqrt{n^{2} \pi^{2}\left(\frac{1-e}{1+e}\right)^{2}+1}
$$

the left-hand side inequality corresponds to the physical limit where the maximum energy the edges can deliver is equal to the dissipated energy. Each periodic orbit depicted in Table I is composed of two phase-locked period-one bouncing balls.

Equation (43) holds under a "small-amplitude" assumption which has been studied in [8]. The small-amplitude assumption of the bouncing ball assumes that the edges motion is much smaller that the ball motion, the impacts therefore occurring at a constant position. Significant differences between the exact and the approximated models are more likely if $e$ is below about 0.8 ; nevertheless, the approximated model provides a good description of the qualitative dynamical behavior.

\section{B. The General Vibrating Wedge-Billiard}

The dynamics of a ball in the general wedge-billiard do not decouple in two independent dynamics along each edge. The frequency-locking relation (37) between two successive impact times on the same edge is still valid. In contrast, we introduce a scaling parameter $\rho$ in (38) to take the energy dissymmetry into account

$$
\Delta t^{r_{*}}=(2 m-1) \rho \frac{\pi}{\omega}
$$

The parameter $\rho$ must fulfill $0<\rho<(2 n / 2 m-1)$, tuning the flight time $\Delta t^{r_{*}}$ between 0 and $(t[k+2]-t[k])^{*}$.

Injecting the flight-time solutions (30) in (37), we obtain a relation on the mean of the normal velocities

$$
\left|V_{n}{ }^{r_{*}}\right|+\left|V_{n}{ }^{l_{*}}\right|=\frac{\pi n g}{\omega} \frac{1+\alpha^{2}}{\alpha^{2}}
$$

that must be combined with (44) to obtain the steady-state normal velocities of the periodic orbits, while the energy levels, the radial velocities, and the impact position derive from (27)-(29)

$$
\begin{aligned}
E^{r_{*}} & =\frac{1}{2 \alpha^{2}}\left(\frac{n \pi g}{\omega}\right)^{2}\left(\left(\frac{(2 m-1) \rho}{2 n}\right)^{2}\left(\alpha^{2}-1\right)+1\right) \\
E^{l_{*}} & =\frac{1}{2 \alpha^{2}}\left(\frac{n \pi g}{\omega}\right)^{2}\left(\left(\frac{2 n-(2 m-1) \rho}{2 n}\right)^{2}\left(\alpha^{2}-1\right)+1\right) \\
\left|V_{n}{ }^{r_{*}}\right| & =\frac{n \pi g}{\omega} \frac{\left(\alpha^{2}-1\right) \frac{(2 m-1) \rho}{2 n}+1}{\alpha^{2}}
\end{aligned}
$$

$$
\begin{aligned}
\left|V_{n}{ }^{l_{*}}\right| & =\frac{n \pi g}{\omega} \frac{\left(1-\alpha^{2}\right) \frac{(2 m-1) \rho}{2 n}+\alpha^{2}}{\alpha^{2}} \\
V_{r}{ }^{r_{*}} & =\frac{-\pi g}{\omega}(n-(2 m-1) \rho)=-V_{r}{ }^{l_{*}} \\
R^{*} & =\frac{g\left(1+\alpha^{2}\right)}{4 \alpha^{2}}\left(\frac{\pi}{\omega}\right)^{2}(2 m-1) \rho(2 n-(2 m-1) \rho) .
\end{aligned}
$$

The energy exchange between the ball and the edges at each impact is

$$
\pm\left(E^{l_{*}}-E^{r_{*}}\right)= \pm \frac{\alpha^{2}-1}{2 \alpha^{2}}\left(\frac{n \pi g}{\omega}\right)^{2}\left(\frac{n-(2 m-1) \rho}{n}\right) .
$$

In the bouncing-ball dynamics, the bifurcation parameter is a nondimensional amplitude proportional to the vibration amplitude $A$ and to the square of the vibration frequency $\omega^{2}$ [6]. Therefore, both these parameters can be used to generate the cascade of bifurcations. This nondimensional parameter depends on $\rho$. This rests on a trigonometric relation between the steady-state edges phases at impact, deriving from $\sin ^{2} \phi^{r_{*}}+\cos ^{2} \phi^{r_{*}}=1$

$$
\begin{array}{r}
\cos ^{2} \phi^{r_{*}}+\cos ^{2} \phi^{l_{*}}-2 \cos \phi^{r_{*}} \cos \phi^{l_{*}} \cos ((2 m-1) \rho \pi) \\
=\sin ^{2}((2 m-1) \rho \pi)
\end{array}
$$

which is not invertible.

Summarizing the derivations in this section, we obtain the following proposition.

Propostion 3: The wedge-billiard model (3) with harmonic actuation $\tilde{s}(t)=A \sin (\omega t)$ isolates particular periodic orbits among those derived in Proposition 2. These orbits are characterized by (46)-(51) via $n$ and $m$, and satisfy frequency-locking relations between the ball and the edges [(37) and (44)]. For the particular square wedge configuration $\left(\theta=45^{\circ}\right)$, (44) degenerates to (38). Considering a cartoon model for a sinusoidal actuation (the parallel actuation depicted in Fig. 5), the dynamics decouple into two 1-DOF dynamics along each edge. Stability of the isolated periodic orbits follows immediately from the bouncing-ball stability (43).

\section{Stabilization of Periodic Orbits by ROTATIONAL ACTUATION OF THE EDGES}

For practical reasons, the experimental setup presented in Section VI uses rotational actuation of the edges instead of linear actuation, such as is depicted in Fig. 5. We examine in this section how rotational actuation modifies the model studied in the previous sections, both for period-one and period-two orbits.

With rotational actuation, the angle $\theta$ of each edge with the vertical is no longer constant, which significantly complicates the derivation of the flight map. To avoid the complication of computing that new flight map, a "small-amplitude" assumption is introduced. We neglect the variation of $\theta$ in the derivation of the flight map, but only take it into account in the derivation of the impact map. As illustrated in Fig. 6, this simplification amounts to assuming that the impacts always occur at angle $\theta$, but that the angular actuation $\mu$ rotates the normal and tangential directions of the impacted edge by an angle $\mu$ (Fig. 6, right). This 

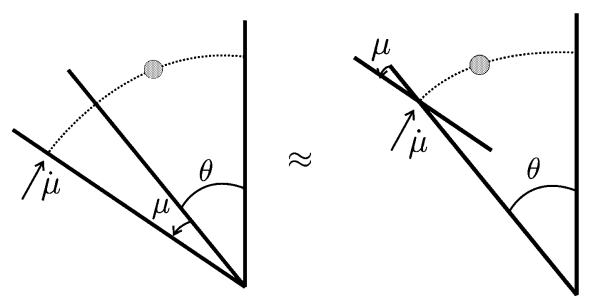

Fig. 6. Controlled rotational wedge (left), and the simplified model when $\mu$ is small (right).

simplification neglects the displacement of the impact point, and is more likely if $|\mu| \ll \theta$.

The Poincaré map of the rotational wedge is derived in Appendix I.

The general steady-state equations derived in Section IV still hold in that case [(46)-(51)]. The edges velocity being equal to $\dot{\mu}(t)=A \omega \cos (\omega t)$, the steady-state edges phases derive from (48), (49), and (59)

$\phi^{r_{*}}=\arccos \left(\frac{1-e}{1+e} \frac{n \pi g}{A R^{*} \omega^{2}} \frac{\left(\alpha^{2}-1\right) \frac{(1+e) \frac{(2 m-1) \rho}{2 n}-e}{1-e}+1}{\alpha}\right)$

$\phi^{l_{*}}=\arccos \left(\frac{1-e}{1+e} \frac{n \pi g}{A R^{*} \omega^{2}} \frac{\left(\alpha^{2}-1\right) \frac{(1+e) \frac{(2 m-1) \rho}{2 n}-1}{1-e}-1}{\alpha}\right)-\pi$.

Because the steady-state impact position $R^{*}$ is proportional to $\left(1 / \omega^{2}\right)(51)$, the nondimensional bifurcation parameter is now only proportional to the vibration amplitude $A$. The vibration frequency acts as a temporal scaling factor, and does not influence the stability properties.

To analyze how the modified model affects the stability of periodic orbits, we numerically computed the eigenvalues of the linearized Poincaré map in two simple cases:

- the period-one orbits $(n=2 m-1, \rho=1)$;

- the period-one and period-two orbits in the square rotational wedge $\left(\theta=45^{\circ}, \rho=1\right)$.

Stability of Period-One Orbits: The simplest period-one orbit $(n=m=1)$ has been first mentioned as an open-loop stable orbit in a wedge-billiard with a rotational sinusoidal actuation in [5]. The stability region presented in this paper is in agreement with our results. This stability region has been obtain from a linearized model which does not rest on a "small-amplitude" assumption. Accordance between stability regions obtained from both models is viewed as a validation of our "small-amplitude" model.

The period-one orbits are particular cases of (46)-(51) and (53) with $n=2 m-1$ and $\rho=1$, which is required for periodone. We find

$$
\begin{aligned}
E^{*} & =\frac{3+\alpha^{2}}{8 \alpha^{2}}\left(\frac{(2 m-1) \pi g}{\omega}\right)^{2} \\
\left|V_{n}^{*}\right| & =\frac{1+\alpha^{2}}{\alpha^{2}} \frac{(2 m-1) \pi g}{2 \omega} \\
V_{r}^{*} & =0 \\
R^{*} & =\frac{1+\alpha^{2}}{4 \alpha^{2}}\left(\frac{(2 m-1) \pi}{\omega}\right)^{2} g
\end{aligned}
$$

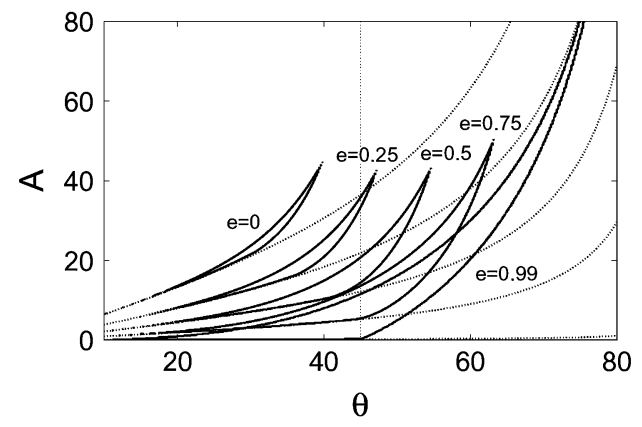

(a)

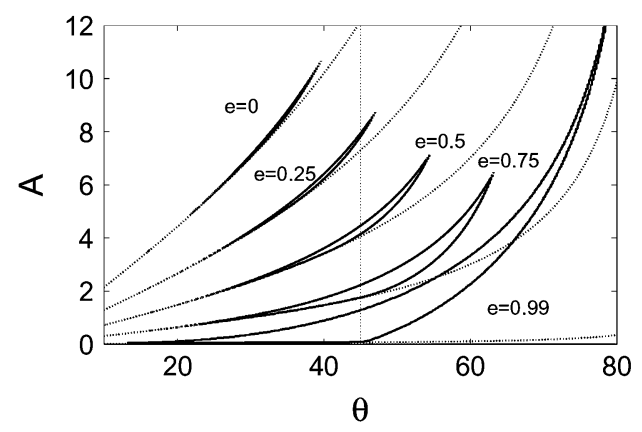

(b)

Fig. 7. Parametric stability region of two period-one orbits in the general wedge (solid lines). The dotted lines denote the physical minimum value for the amplitude $A$ (the arccos argument in (58) must be $\leq 1$ ).

$$
\phi^{*}=\arccos \left(\frac{1-e}{1+e} \frac{2 \alpha}{A} \frac{1}{(2 m-1) \pi}\right) .
$$

The stability is studied via the linearized Poincaré map of $\tilde{\mathcal{B}}$ (59) and of the flight time (5) around the period-one solution just derived. We find the Jacobian matrix (65) derived in Appendix II. Its eigenvalues were numerically computed for several values of $e$ and $\alpha$. Fig. 7 depicts the stability region for the first two period-one orbits [(a) $n=1, m=1$ and (b) $n=3, m=2]$. The superposed curves stand for different values of $e$.

A decreasing coefficient of restitution reduces the stability regions and shifts it in a zone corresponding to closer angles between the edges. We can see that even for $e=0$, the first period-one orbit is still theoretically stabilizable with a sinusoidal vibration of the edges, if the impacts occur with $\theta \in\left[15^{\circ}, 40^{\circ}\right]$.

We conclude that sinusoidal actuation of edges stabilizes period-one orbits for any coefficient of restitution $e<1$ and for a broad domain of wedge geometry. For $\theta>45^{\circ}$, this exponential stability is in sharp contrast with the instability of the same periodic orbit in the fixed elastic wedge.

Stability of Period-Two Orbits in the Square Wedge: With linear actuation of the edges, we have shown in Section IV-A that stability of period-two orbits in the square wedge follows from the bouncing-ball dynamics analysis, yielding exponentially stable orbits in the parameter range (43). We now show that this stabilization result also holds with rotational actuation of the edges, by computing the eigenvalues of the Jacobian linearization of the map $\tilde{\mathcal{B}} \circ \tilde{\mathcal{B}}=\tilde{\mathcal{B}}^{2}(59)$ and of (5) around the period-two solution. We find the matrix $\tilde{\mathcal{M}}_{2} \tilde{\mathcal{M}}_{1}$ derived in Appendix II, equation (67). 


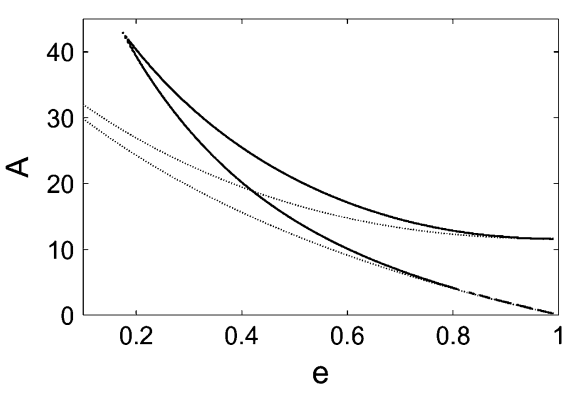

(a)

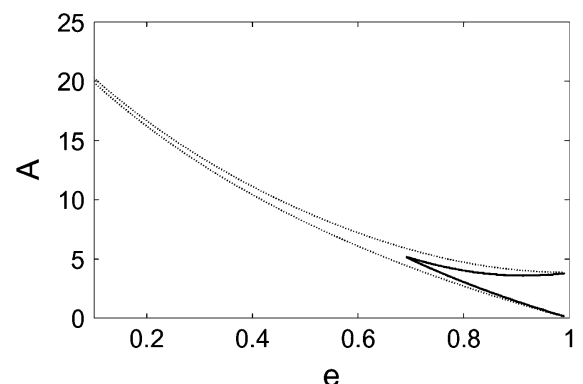

(b)

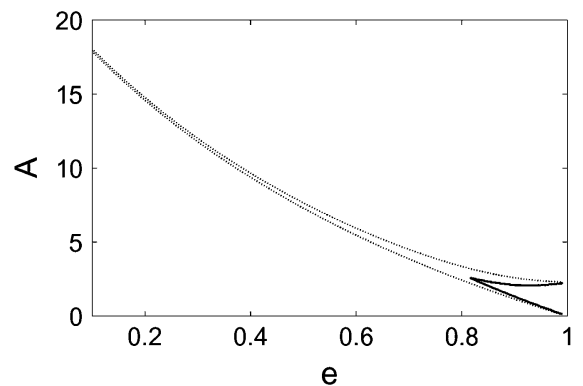

(c)

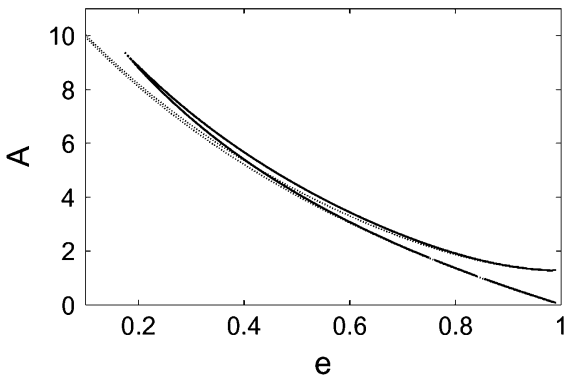

(d)

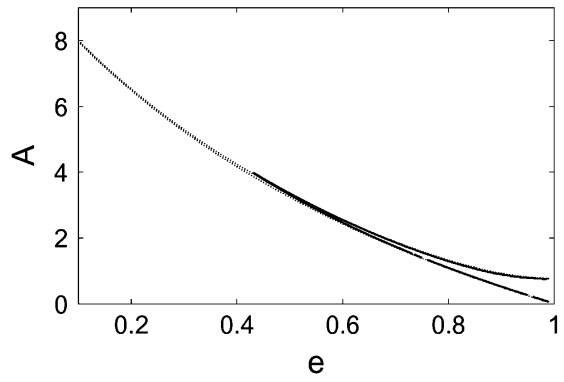

(e)

Fig. 8. Parametric stability region of periodic orbits in the square wedge with linear actuation (dotted) and rotational actuation (solid). (a) $n=1, m=1,(1: 1)$. (b) $n=2, m=1,(1: 3)$. (c) $n=3, m=1,(1: 5)$. (d) $n=3, m=2,(1: 1)$. (e) $n=4, m=2,(3: 5)$.

Despite the new dynamics of the rotational wedge, there still exists a region in the parameters space where the absolute values of the four eigenvalues of this matrix are less than one. This region is depicted in Fig. 8 (solid lines) for five of the periodic solutions emphasized. $(n=1, m=1)$ and $(n=3, m=2)$ are two (1:1) period-one solutions depicted in Fig. 8(a) and (d), respectively, while $n=2, m=1,(1: 3) ; n=3, m=1$, (1:5); and $n=4, m=2,(3: 5)$ are three period-two solutions depicted in Fig. 8(b), (c), and (e), respectively. Each of those stability regions is compared with the corresponding stability region of the double bouncing-balls system (dotted lines), where $\sqrt{2} A$ has been replaced by $A R^{*}$ in (43). These stability regions clearly overlap more accurately for a high coefficient of restitution $e$. This makes physical sense: the larger the coefficient of restitution, the smaller the vibration, and thus the more acceptable the small-angle assumption.

Note that each of the curves depicted in Fig. 7 crosses the $\theta=45^{\circ}$ line (light dotted) with intervals corresponding to those drawn in Fig. 8(a) and (d), for the corresponding values of $e$.

Stability of Period-Two Orbits in the Actuated Nonsquare Wedge: The eigenvalues of the Jacobian matrix of the period-two orbits in the nonsquare wedge have not been computed. By analogy with the period-one orbits, we expect the conclusions obtained for the square wedge to persist in a range of values around $\theta=45^{\circ}$.

The results of this section are summarized in the following proposition.

Propostion 4: For a broad range of parameters $(\theta, e, A)$, a sinusoidal actuation of the edges around a common fixed point (i.e., rotational actuation) achieves exponential stability of isolated periodic orbits. In particular, Fig. 7 illustrates the parametric stability region of two period-one orbits, and Fig. 8 illustrates the parametric stability region of five periodic orbits in the square configuration.

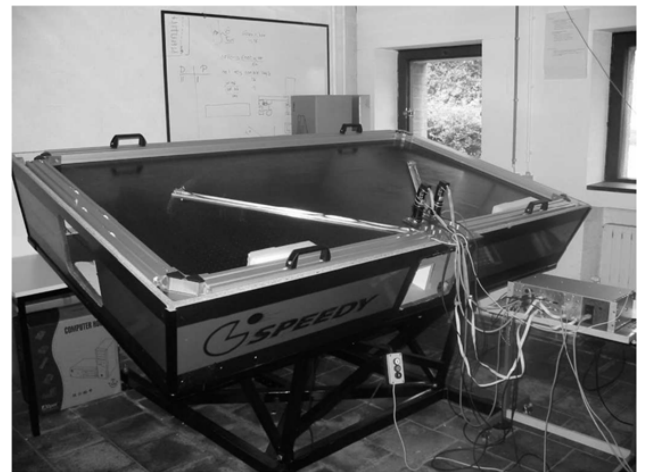

Fig. 9. Picture of Wiper.

\section{EXPERIMENTAL RESULTS}

This section describes an experimental validation of the stability results derived in the previous section for period-one and period-two orbits. ${ }^{3}$

Wiper is a planar juggling robot designed from the model of the rotational wedge, both edges being actuated around their fixed point. Fig. 9 is a picture of this robot, which is called "Wiper" by analogy with the periodic rotative motion of windscreen wipers. The motion plane is a tilted air-hockey table. The gravity field $\mathrm{g}$ can be adjusted by proper inclination of the table. This acts as a tempo scale factor in the fixed-points equations. This table is pierced with a lattice of little holes, separated about $4 \mathrm{~cm}$ from each other. These holes blow air constantly from the table in order to provide a frictionless motion of the sliding bodies. The sliding body is a small plastic puck.

${ }^{3}$ The interested reader will find two movies illustrating the stabilization of these periodic orbits on http://ieeexplore.ieee.org or on the first author's homepage. 
Two metallic edges were mounted on the rigid frame of the table. Both of them are controlled by an independent motor, as close as possible to each other in order to match their fixed intersection in the model. The edges motors are controlled with a real-time computer running with XPCTARGET (The Mathworks). We measured the impact times, detected by two accelerometers mounted at the edges' top points, in order to check the tempo of the stabilized orbits.

The model we derived throughout the previous sections differs clearly from the actual setup. These differences are covered by the following mechanical assumptions:

1) the edges are not affected by the impacts, so that their velocity is continuous at impact times;

2) the impacts are localized in space around the fixed wedge ("small angle" assumption);

3) the contact is frictionless.

Assumption 1 relies on the fact that the edges are largely heavier than the puck. Because the edges' motors track a sinusoidal reference signal, the perturbation caused at one impact time is rejected by the next impact time. Note that a finite mass ratio between the actuator and the object can be captured by a modified coefficient of restitution; see, e.g., [22] for a 1-D bouncing ball model capturing this effect. The effect of Assumption 2 is more tedious to analyze. Relaxing the "small angle" assumption leads to an implicit billiard map that is much more complicated to solve. Assumption 3 is reasonable. Friction at impact induces the puck to spin. This leads to a more complicated model that will have topologically similar steady-state orbits. See the discussion in Section VI-B. The impact controllability of an air hockey puck has been studied in [23].

The goal of the crude model we derived is to capture the main effect of sensorless actuation of a wedge-billiard. The good matching between our experimental data and the theoretical predictions (see the rest of this section) is the best argument we can provide to validate the model.

\section{A. Stabilization of Period-One Orbits}

Our first experimental result is the stabilization of the first period-one orbit $(n=m=1)$ with rotational sinusoidal actuation of the edges. According to Fig. 7(a), two parameters are supposed to be crucial in the determination of the theoretical stability region: $\alpha$, which is a geometrical parameter of the wedge and is therefore derived from the edges position, and the coefficient of restitution $e$, which, on the contrary, is difficult to estimate.

For a set of initial wedge apertures, we experimentally isolated the amplitude domains where the first period-one motion is stable. The experiments were conducted with a table inclined at an angle of $15^{\circ}$ and a vibration frequency tuned in order to have a steady-state impact position $R^{*}$ close to $0.7 \mathrm{~m} \mathrm{[(57)].}$ Both parameters serve as tempo scaling factors and do not influence the stability regions.

Fig. 10 exhibits the experimental results obtained: all the tested conditions are marked by a point. The white and light gray zones are simply crude "contour" interpolations of the observed stability regions: all experimentally stable conditions belong to the white set. The light gray set contains the

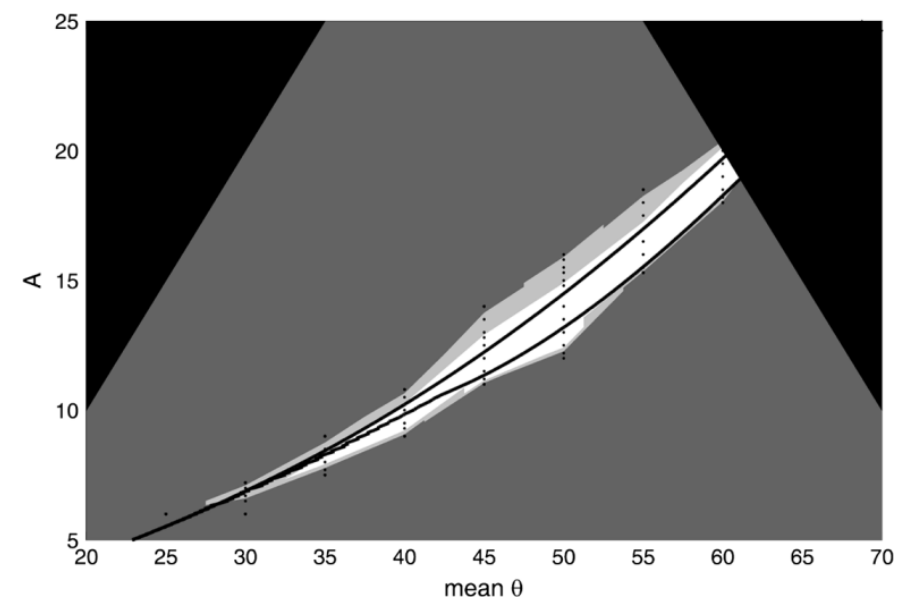

Fig. 10. Experimental results on period-one stabilization: the points correspond to the tested conditions, the white zone surrounds the stability region, the light gray zone surrounds the "weak stability" region (see text), the dark gray zone depicts the instability region, and the black zone excludes the nonsecure zone. The results are compared with the theoretical prediction (solid lines) for $e=0.5$.

conditions which have been sorted as fragile but stable, in the sense that either the period-one was stable, but only for a limited number of impacts (basically more than 10, but less than 30), or the puck described a complex motion confined around the period-one solution. The dark gray zone covers the experimental instability region, and the black zone excludes the experimental conditions leading to a risk of collision between the edges or with the table frame.

In order to compare the experimental results with the theoretical predictions, we superposed the theoretical stability region (solid lines) on Fig. 10, for $e=0.5$. This region fits the experimental results very well, and therefore validates the model and the mathematical analysis presented in the previous sections.

We note that Figs. 10 and 7(a) should be compared with caution. In Fig. 10, the parameter along the horizontal axis is the mean edge angle, which is easily determined experimentally. However, the mean edge angle differs from the impact angle, used as a parameter in Fig. 7. This explains why the theoretical parameter stability region in Fig. 10 appears to be shifted and elongated, compared with the corresponding region in Fig. 7(a).

\section{B. Stabilization of Period-Two Orbits}

Experimental stabilization of period-two orbits turned out to be much more challenging. This is partly explained by the reduced stability regions of period-two orbits (see Fig. 8), but we also point out two additional reasons supporting that fact.

- The basins of attraction of the period-two orbits are much smaller than the period-one ones. A period-two orbit will be stabilized with a faster vibration frequency than a period-one orbit with an equivalent steady-state impact position (compare the $\omega$ obtained from (51) and (57) for similar values of $R^{*}$ ). The period-two orbits are, therefore, more sensitive to the phase initial condition.

- The nonzero tangential impact velocity in period-two orbits causes the puck to spin. This phenomenon, not captured by the model, likely affects the stability properties, and requires further investigations. 


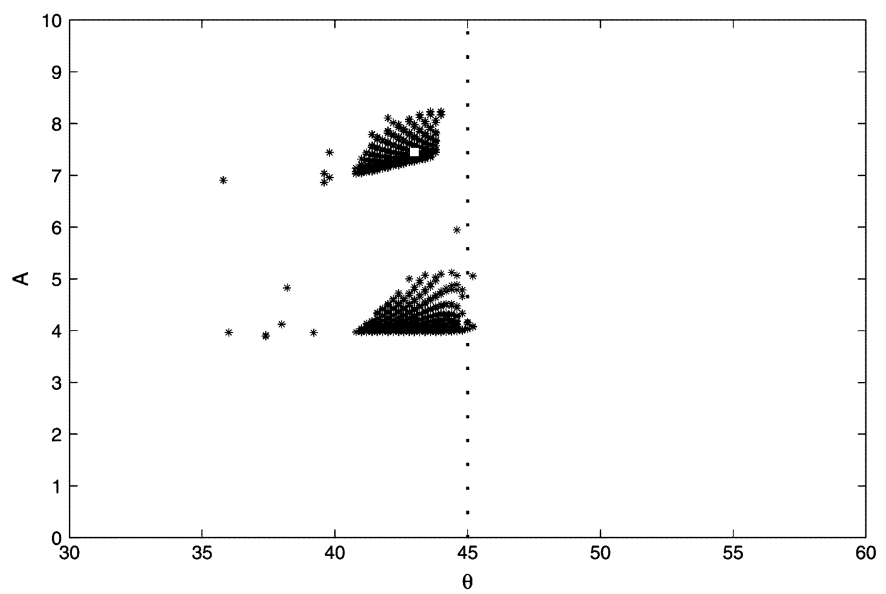

Fig. 11. Stability region of the $(n=2, m=1)$ period-two orbit in the general Wiper for $e=0.5$ (black points) and $e=0.7$ (gray points).

We focused on the stabilization of the first period-two orbit $(n=2, m=1)$. Fig. 8(b) shows that this periodic solution is not stable in the square configuration for the expected coefficient of restitution $(e \approx 0.5)$. The Jacobian matrix of the period-two orbits in the general Wiper being hard to calculate, we preferred to numerically simulate the effect of small perturbations in our dynamical model, around the period-two limit cycle.

The points lying on Fig. 11 correspond to parameter values where a small perturbation of the period-two solution decreases through the impacts. These points give an idea of the parametric stability region of that period-two orbit. Fig. 11 is consistent with Fig. 8(b): ${ }^{4}$ the parametric stability region has no intersection with the $45^{\circ}$ black dotted line for $e$ smaller than 0.7 .

We successfully stabilized the period-two orbit in the lab during more than 50 impacts with a vibration amplitude $A$ equal to $7.45^{\circ}$. The impact angular position has been visually estimated around $43^{\circ}$. This point is marked by a white square in Fig. 11, and clearly belongs to the numerically predicted stability region of the $(n=2, m=1)$ period-two orbit. This result opens the door to the stabilization of juggling patterns implying several pucks (two in this case), requiring only a temporal separation of the pucks equal to $(2 \pi / \omega)$. In this way, Wiper would mimic the popular shower juggling pattern.

\section{Conclusion AND PERSPECTIVES}

This paper has established the experimental validation of the sensorless stabilization of bounce juggling patterns. This result is also supported by a mathematical analysis of the modified stability properties. From periodic orbits which have been proved to be unstable, we derived an actuation law which stabilizes the same patterns. The wedge-billiard has been used to illustrate the potential of sensorless stabilization in rhythmic tasks. Section II described its dynamics and derived its model. We considered the proposed sensorless strategy as a useful control method for this kind of rhythmic task, certainly sacrificing some performance in robustness, but avoiding the costs pertaining to

${ }^{4}$ Let us recall that Figs. 8(b) and 11 slice the stability region differently in the 3-D parameter space $(e, \theta, A)$ : Fig. 8 (b) slices the parameter space for $\theta=45^{\circ}$, while Fig. 11 slices the parameter space for $e=0.5$ and $e=0.7$. These figures match at their intersections, $\left(\theta=45^{\circ}, e=0.5\right)$ and $\left(\theta=45^{\circ}, e=0.7\right)$. sensor development. Aiming at mimicking the popular shower juggling pattern, in Section III, two particular periodic solutions of the wedge-billiard were studied: the period-two orbit and its degenerate case, i.e., the period-one orbit. These periodic solutions have been proved to be either marginally stable or unstable for an uncontrolled elastic wedge-billiard. In Section IV, we derived the steady-state periodic orbits of a periodically actuated wedge-billiard. For the particular square wedge-billiard configuration, this generalizes the results on the bouncing-ball dynamics [6] to stabilize the wedge-billiard periodic orbits. A practical implementation of this stabilization requires a different configuration, which has been modeled in Section V. Finally, these theoretical predictions on the stabilization of periodic orbits of the wedge-billiard have been validated by several experimental results on the Wiper robot, and are explained in Section VI. Fig. 10 emphasized that our model underestimated the stability region for the first period-one orbit. The dynamical properties that are not captured by our model seem to have a stabilizing effect on the period one.

Future work will aim at applying these results to human behavior modeling. This is, for example, of relevant interest, to investigate the human abilities to exploit the open-loop stability of the periodic orbits with a sinusoidal actuation, when the subject is asked to control one of these orbits. Open-loop and closedloop balance could be measured with the "negative acceleration" criterion [9]. The role of feedback sources, and the strategy which is employed to select the points of relevant (visual) information can also be investigated with this setup.

Equations related to the appendixes:

$$
\begin{aligned}
& \left(\begin{array}{c}
V_{r}[k+1] \\
V_{n}[k+1]
\end{array}\right) \\
& =J(\mu[k+1])\left(\begin{array}{c}
\left|V_{n}\right|[k]-V_{r}[k]-\left|V_{n}^{-}\right|[k+1] \\
\left|V_{n}^{-}\right|[k+1] \cdot \operatorname{sign}\left(V_{n}[k]\right)
\end{array}\right) \\
& \quad+\frac{1+e}{\alpha}\left(\begin{array}{c}
-\alpha \sin \mu[k+1] R[k+1] \\
\cos \mu[k+1] R[k+1]
\end{array}\right) \dot{\mu}[k+1] \\
& R[k+1] \\
& =R[k]+\frac{1}{2 g} V_{r}^{2}[k]+\frac{\alpha^{2}}{2 g} V_{n}^{2}[k]-\frac{1}{2 g}\left(\left|V_{n}\right|[k]-V_{r}[k]\right. \\
& \left.\quad-\left|V_{n}^{-}\right|[k+1]\right)^{2}-\frac{\alpha^{2}}{2 g}\left|V_{n}^{-}\right|^{2}[k+1]
\end{aligned}
$$

with (60)-(62), shown at the top of the next page.

\section{APPENDIX I \\ ITERATION MAP OF THE ROTATIONALLY ACTUATED WEDGE-BILLIARD}

Under the "small amplitude" assumption (Fig. 6), the impact rule $\mathcal{I}$ captures the angular rotation. It is now given by

$$
\begin{aligned}
M(\mu[k])\left(\begin{array}{l}
V_{r}[k] \\
V_{n}[k]
\end{array}\right)=\left(\begin{array}{cc}
1 & 0 \\
0 & -e
\end{array}\right) & M(\mu[k])\left(\begin{array}{c}
V_{r}^{-}[k] \\
V_{n}^{-}[k]
\end{array}\right) \\
& +\left(\begin{array}{c}
0 \\
\frac{1+e}{\alpha} R[k]
\end{array}\right) \dot{\mu}[k]
\end{aligned}
$$

with $M(\mu)$ denoting the rotation matrix of the edge

$$
M(\mu)=\left(\begin{array}{cc}
\cos \mu & \alpha \sin \mu \\
-\frac{\sin \mu}{\alpha} & \cos \mu
\end{array}\right)
$$




$$
\begin{aligned}
& J(\mu)=M(-\mu)\left(\begin{array}{cc}
1 & 0 \\
0 & -e
\end{array}\right) M(\mu)=\left(\begin{array}{cc}
\cos ^{2} \mu-e \sin ^{2} \mu & \frac{\alpha(1+e)}{2} \sin 2 \mu \\
\frac{1+e}{2 \alpha} \sin 2 \mu & \sin ^{2} \mu-e \cos ^{2} \mu
\end{array}\right) \\
& \tilde{\mathcal{M}}=\left(\begin{array}{cccc}
\frac{1-4 \alpha^{2}-\alpha^{4}}{\left(1+\alpha^{2}\right)^{2}}-2 \mu_{11} & \frac{4 \alpha^{2}}{\left(1+\alpha^{2}\right)^{2}}-\left(\alpha^{2}-1\right) \mu_{11} & -\frac{4 \alpha^{2}}{(2 m-1)\left(1+\alpha^{2}\right)^{2}}-\frac{2}{(2 m-1)} \mu_{11} & -\frac{1-e}{1+e} 2\left(1+\alpha^{2}\right) \\
\frac{2 e\left(\alpha^{2}-1\right)}{\left(1+\alpha^{2}\right)^{2}}+(2 m-1) \mu_{21} & \frac{e\left(\alpha^{2}-1\right)^{2}}{\left(1+\alpha^{2}\right)^{2}}+\mu_{22} & \frac{4 e \alpha^{2}}{(2 m-1)\left(1+\alpha^{2}\right)^{2}}+\mu_{21} & -\frac{(1-e)\left(1+\alpha^{2}\right)(2 m-1)^{2} \Gamma^{\prime}}{4 \alpha^{3}} \\
\frac{(2 m-1)\left(1-\alpha^{2}\right)}{1+\alpha^{2}} & \frac{2(2 m-1) \alpha^{2}}{1+\alpha^{2}} & \frac{1-\alpha^{2}}{1+\alpha^{2}} & 0 \\
\frac{4 \alpha^{2}}{\left(1+\alpha^{2}\right)^{2}} & \frac{2 \alpha^{2}\left(\alpha^{2}-1\right)}{\left(1+\alpha^{2}\right)^{2}} & \frac{4 \alpha^{2}}{(2 m-1)\left(1+\alpha^{2}\right)^{2}} & 1
\end{array}\right) \\
& \mu_{11}=\frac{1-e}{1+e} \frac{4 \alpha^{2}}{1+\alpha^{2}} \\
& \mu_{21}=(1-e)\left(\frac{-(2 m-1) \Gamma^{\prime}}{\alpha\left(1+\alpha^{2}\right)}+\frac{2\left(1-\alpha^{2}\right)}{(2 m-1)\left(1+\alpha^{2}\right)}\right) \\
& \mu_{22}=(1-e)\left(\frac{\left(1-\alpha^{2}\right)(2 m-1)^{2} \Gamma^{\prime}}{2 \alpha\left(1+\alpha^{2}\right)}+\frac{4 \alpha^{2}}{1+\alpha^{2}}\right) \\
& \Gamma^{\prime}=\sqrt{\left(\left(\frac{1+e}{1-e}\right) A \pi^{2}\right)^{2}-\left(\frac{2 \pi \alpha}{2 m-1}\right)^{2}} \\
& \tilde{\mathcal{M}}_{1}=\left(\begin{array}{cccc}
-\frac{2 \eta}{\eta+\nu}-\frac{1-e}{1+e} \frac{2(\eta+\nu)}{\nu} & 1 & -\frac{2}{\eta+\nu}-\frac{1-e}{1+e} \frac{2(\eta+\nu)}{\eta \nu} & -\frac{1-e}{1+e} \frac{(\eta+\nu)^{2}}{\eta \nu} \\
\frac{-e(\nu-\eta)}{\eta+\nu}+(1-e)\left(\frac{2 \eta \beta}{\eta+\nu}+\frac{\nu-\eta}{\nu}\right) & (1-e) \frac{\eta+\nu}{\nu} & \frac{2 e}{\eta+\nu}+(1-e)\left(\frac{2 \beta}{\eta+\nu}+\frac{\nu-\eta}{\eta \nu}\right) & (1-e) \beta \\
\frac{(\nu-\eta) \eta}{\eta+\nu} & \eta & \frac{\nu-\eta}{\eta+\nu} & 0 \\
\frac{2 \eta}{\eta+\nu} & 0 & \frac{2}{\eta+\nu} & 1
\end{array}\right) \\
& \beta=\frac{(\nu+\eta)(\eta-\nu)}{\nu \eta}-\frac{\nu \eta}{2} \sqrt{\left(\left(\frac{1+e}{1-e}\right) A \pi^{2}\right)^{2}-\left(\frac{2 n \pi}{(2 m-1)(2 n-2 m+1)}\right)^{2}}
\end{aligned}
$$

and $\mu[k]=\mu(t[k])(\dot{\mu}[k]=\dot{\mu}(t[k]))$ denoting the edge position (velocity) at impact time $t[k]$. The presence of $R[k]$ in (63) is due to a second important feature introduced by the rotational actuation. The energy exchange with the edges depends now on the impact position $R[k]$. The map $\tilde{\mathcal{B}}$ of the rotational wedge is given by (59). The flight time is still given by (5).

The "small amplitude" assumption is done around the steadystate periodic orbits. They are, therefore, unchanged with respect to those which have been derived in Section IV. The actual actuation law is then

$$
\mu(t)=A\left(\sin \omega t-\sin \left(\left\{\phi^{r_{*}}, \phi^{l_{*}}\right\}\right)\right)
$$

providing $\mu^{*}=0$.

\section{APPENDIX II}

\section{JACOBIAN MATRICES}

The linearized Poincaré map of the period-one orbits of the rotational wedge-billiard is the matrix $\tilde{\mathcal{M}}$

$$
\left(\begin{array}{c}
\frac{\omega}{g} \delta V_{r}[k+1] \\
\frac{\omega}{g} \delta\left|V_{n}\right|[k+1] \\
\frac{\omega^{2}}{\pi g} \delta R[k+1] \\
\omega t[k+1]
\end{array}\right)=\tilde{\mathcal{M}}\left(\begin{array}{c}
\frac{\omega}{g} \delta V_{r}[k] \\
\frac{\omega}{g} \delta\left|V_{n}\right|[k] \\
\frac{\omega^{2}}{\pi g} \delta R[k] \\
\omega t[k]
\end{array}\right)
$$

that is given in (61).

Note that the determinant of $\tilde{\mathcal{M}}$ is equal to

$$
|\tilde{\mathcal{M}}|=\frac{e\left((1-e)\left(3 \alpha^{2}-1\right)+2\left(1+\alpha^{2}\right)\right)}{(1+e)\left(1+\alpha^{2}\right)}
$$

which is equal to one in the elastic case $(e=1) \forall \alpha$. This illustrates that the sinusoidal input does not achieve exponential stability of the period-one orbit in the elastic wedge, because all the eigenvalues of this matrix cannot be $<1$ in that case.

The linearized Poincaré map of the periodic orbits of the rotational square wedge-billiard is the matrix $\tilde{\mathcal{M}}_{2} \tilde{\mathcal{M}}_{1}$

$$
\begin{array}{r}
\left(\begin{array}{c}
\frac{\omega}{g} \delta V_{r}[k+2] \\
\frac{\omega}{g} \delta\left|V_{n}\right|[k+2] \\
\frac{\omega^{2}}{\pi g} \delta R[k+2] \\
\omega t[k+2]
\end{array}\right) \\
=\tilde{\mathcal{M}}_{2}\left(\begin{array}{c}
\frac{\omega}{g} \delta V_{r}[k+1] \\
\frac{\omega}{g} \delta\left|V_{n}\right|[k+1] \\
\frac{\omega^{2}}{\pi g} \delta R[k+1] \\
\omega t[k+1]
\end{array}\right) \\
=\tilde{\mathcal{M}}_{2} \tilde{\mathcal{M}}_{1}\left(\begin{array}{c}
\frac{\omega}{g} \delta V_{r}[k] \\
\frac{\omega}{g} \delta\left|V_{n}\right|[k] \\
\frac{\omega^{2}}{\pi g} \delta R[k] \\
\omega t[k]
\end{array}\right) .
\end{array}
$$

$\tilde{\mathcal{M}}_{1}$ is given in (62), where $\eta=2 m-1$ and $\nu=2 n-2 m+1$ depends on the steady-state flight times. $\tilde{\mathcal{M}}_{2}$ has exactly the same structure as $\tilde{\mathcal{M}}_{1}$ with an exchange between $\nu$ and $\eta$.

\section{ACKNOWLEDGMENT}

The authors would like to thank M. Gérard for his contributions to the development of Wiper and M. De Wan for his precious technical assistance during the design of this robot. They 
would also like to thank the reviewers for the helpful comments that improved the paper.

\section{REFERENCES}

[1] M. Buehler, D. Koditschek, and P. Kindlmann, "A one degree of freedom juggler in a two degree of freedom environment," in Proc. IEEE/RSJ Conf. Intell. Syst. Robots, Tokyo, Japan, 1988, pp. 91-97.

[2] — "A family of robot control strategies for intermittent dynamical environments," IEEE Control Syst. Mag., vol. 10, no. 2, pp. 16-22, Feb. 1990.

[3] _ , "Planning and control of robotic juggling and catching tasks," Int. J. Robot. Res., vol. 13, no. 2, pp. 101-118, 1994.

[4] U. Saranli, M. Buehler, and D. Koditschek, "Rhex -A simple and highly mobile hexapod robot," Int. J. Robot. Res., vol. 20, no. 7, pp. 616-631, 2001.

[5] S. Schaal and C. G. Atkeson, "Open-loop stable control strategies for robot juggling," in Proc. IEEE Int. Conf. Robot. Autom., vol. 3, 1993, pp. 913-918.

[6] P. J. Holmes, "The dynamics of repeated impacts with a sinusoidally vibrating table," J. Sound Vibration, vol. 84, no. 2, pp. 173-189, 1982.

[7] J. Guckenheimer and P. J. Holmes, Nonlinear Oscillations, Dynamical Systems and Bifurcations of Vector Fields. New York: Springer-Verlag, 1986.

[8] C. Bapat, S. Sankar, and N. Popplewell, "Repeated impacts on a sinusoidally vibrating table," J. Sound Vibration, vol. 108, no. 1, pp. 99-115, 1986

[9] S. Schaal, C. G. Atkeson, and D. Sternad, "One-handed juggling: A dynamical approach to a rhythmic movement task," J. Mot. Behav., vol. 28, no. 2, pp. 165-183, 1996

[10] D. Sternad, M. Duarte, H. Katsumata, and S. Schaal, "Bouncing a ball: Tuning into dynamic stability," J. Exp. Psychol. Human Percept. Perform., vol. 27, no. 5, pp. 1163-1184, 2001.

[11] _ "Dynamics of a bouncing ball in human performance," Phys. Rev. $E$, vol. 6301, no. 1,2001

[12] C. E. Shannon, "Scientific aspects of juggling," in Claude Elwood Shannon: Collected Papers, N. J. A. Sloane and A. D. Wyner, Eds. New York: IEEE Press, 1993, p. 924.

[13] P. J. Beek and A. Lewbel, "The science of juggling," Sci. Amer, vol. 273, no. 5, pp. 92-97, 1995.

[14] P. J. Beek and A. A. M. van Santvoord, "Learning the cascade juggle—A dynamic-systems analysis," J. Mot. Behav., vol. 24, no. 1, pp. 85-94, 1992.

[15] K. M. Lynch and C. K. Black, "Recurrence, controllability, and stabilization of juggling," IEEE Trans. Robot. Autom., vol. 17, no. Apr., pp. 113-124, 2001.

[16] A. Zavala-Rio and B. Brogliato, "On the control of a one degree-offreedom juggling robot," Dyn. Control, vol. 9, no. 1, pp. 67-90, 1999.

[17] H. L. Jin and M. Zacksenhouse, "Oscillatory neural networks for robotic yo-yo control," IEEE Trans. Neural Netw., vol. 14, no. 2, pp. 317-325, Feb. 2003.

[18] R. Sepulchre and M. Gerard, "Stabilization of periodic orbits in a wedge billiard," in Proc. 42nd IEEE Conf. Decision Control, Maui, HI, 2003, pp. $1568-1573$.

[19] M. Gerard and R. Sepulchre, "Stabilization through weak and occasional interactions: A billiard benchmark," in Proc. IFAC NOLCOS, Stuttgart, Germany, 2004, pp. 75-80.

[20] H. E. Lehtihet and B. N. Miller, "Numerical study of a billiard in a gravitational field," Phys. D, vol. 21, no. 1, pp. 93-104, 1986.
[21] R. Ronsse, P. Lefevre, and R. Sepulchre, "Open-loop stabilization of 2D impact juggling," in Proc. IFAC NOLCOS, Stuttgart, Germany, 2004, pp. $1157-1162$.

[22] T. L. Vincent and A. I. Mees, "Controlling a bouncing ball," Int. J. Bifurc. Chaos, vol. 10, no. 3, pp. 579-592, 2000

[23] M. W. Spong, "Impact controllability of an air hockey puck," Syst. Control Lett., vol. 42, no. 5, pp. 333-345, 2001.

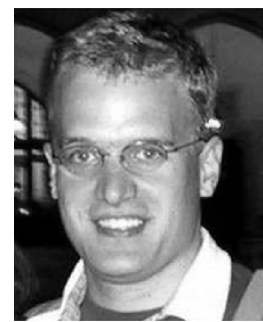

Renaud Ronsse was born in Liège, Belgium, in 1980. He received the University degree in engineering science (electrical engineering) in 2002 from the Université de Liège, Liège, Belgium, where he is currently working toward the Ph.D. degree in the Department of Electrical Engineering and Computer Science.

His thesis topic concerns the behavioral modeling of rhythmic tasks control, both from robotics and human perspectives.

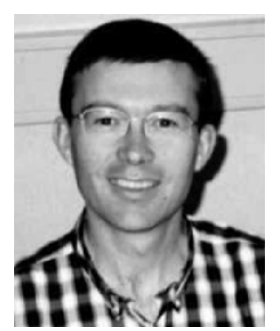

Philippe Lefèvre was born in Ottignies, Belgium, in 1965 . He received the University degree in engineering science (electrical engineering) in 1988, and the Ph.D. degree in engineering in 1992, both from the Université catholique de Louvain (UCL), Louvain-la-Neuve, Belgium. From 1990 to 1991 , he studied as a Ph.D. student in the Department of Biomedical Engineering, McGill University, Montreal, QC, Canada.

He then spent two years (1995-1997) as a Postdoctoral Researcher at the National Institutes of Health (NIH), Bethesda, MD, in the Laboratory of Sensorimotor Research. In 1997, he joined the Fonds National de la Recherche Scientifique (FNRS) of UCL, where he started a new lab, investigating the neural control of movement by combining both experimental and modeling approaches. In 2003-2004, he spent a sabbatical year at the National Eye Institute of the NIH, Bethesda, MD, as a Visiting Scientist.

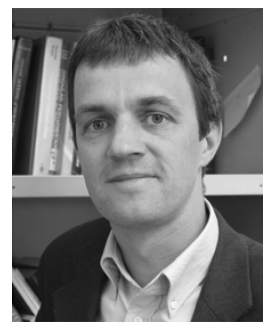

Rodophe Sepulchre (M'96) was born in Brussels, Belgium, in 1967. He received the University and $\mathrm{Ph} . \mathrm{D}$. degrees in engineering science (applied mathematics) from the Université catholique de Louvain, Louvain-la-Neuve, Belgium, in 1990 and 1994, respectively.

From 1994 to 1996, he was a Postdoctoral Research Fellow with the Center for Control Engineering and Computation, University of California, Santa Barbara. He is currently a Professor with the Université de Liège, Liège, Belgium. His research interests lie in control theory, nonlinear dynamical systems, and their applications to problems in mechanical, electrical, and biomedical engineering. He coauthored (with M. Jankovic and P. Kokotovic) the book, Constructive Nonlinear Control (New York: Springer, 1997). 